Supporting information for

\title{
Optical Nature and Binding Energetics of Fluorescent Fluoride Sensor Bis(bora)calix[4]arene and Design Strategies of its Homologues
}

\author{
Jaehyeok Jin ${ }^{1}$, Ji Young Park ${ }^{2}$, and Yoon Sup Lee ${ }^{3}$ \\ Department of Chemistry, Korea Advanced Institute of Science and Technology (KAIST), \\ Yuseong-gu, Daejeon 34141, Korea
}

E-mail: yslee@kaist.edu

1 Present address: Department of Chemistry, The University of Chicago, 5735 South Ellis Avenue, Chicago,
Illinois 60637, United States
2 Present address: Center for Catalytic Hydrocarbon Functionalization, Institute for Basic Science (IBS),
Daejeon 34141 , Korea
3 To whom correspondence should be addressed. Tel: +82-42-350-2821; Fax: +82-42-350-2810 


\section{S1. Calculated ${ }^{1} \mathrm{H}$ NMR shifts of 1}

Calculated ${ }^{1} \mathrm{H}$ NMR chemical shifts (a left column in Table S1) and experimental data ${ }^{1}$ (a right column in Table S1) of bis(bora)calix[4]arene, or 1, are listed in Table S1. These chemical shifts agree with the experimental data to the same degree as other previously reported NMR shift calculations of calixarene derivatives. ${ }^{2,3}$

\section{Table S1. The ${ }^{1}$ H NMR chemical shifts of bis(bora)calix[4] arene using MPW91PW91/6-31G(d,p) (unit: ppm)}

\begin{tabular}{|c|c|c|c|}
\hline \multicolumn{2}{|c|}{ Chemical shift (Degeneracy) } & Cal. & Exp. ${ }^{I}$ \\
\hline Cal. & Exp. ${ }^{T}$ & $7.53(2 \mathrm{H})$ & \\
\hline $8.57(2 \mathrm{H})$ & \multirow{2}{*}{$7.68(4 \mathrm{H})$} & $7.34(2 \mathrm{H})$ & \\
\hline $8.50(2 \mathrm{H})$ & & $5.49(2 \mathrm{H})$ & $5.01(2 \mathrm{H})$ \\
\hline $8.11(2 \mathrm{H})$ & \multirow{2}{*}{$7.22(4 \mathrm{H})$} & $4.23(2 \mathrm{H})$ & $3.89(2 \mathrm{H})$ \\
\hline $7.89(2 \mathrm{H})$ & & $3.56(2 \mathrm{H})$ & $3.47(2 \mathrm{H})$ \\
\hline $7.62(2 \mathrm{H})$ & $7.03(2 \mathrm{H})$ & $3.37(2 \mathrm{H})$ & $3.36(2 \mathrm{H})$ \\
\hline $\begin{array}{c}7.57 \sim \\
7.56(4 \mathrm{H})\end{array}$ & $6.93(4 \mathrm{H})$ & $\begin{array}{c}1.94 \sim \\
1.18(36 \mathrm{H})\end{array}$ & $0.82(36 \mathrm{H})$ \\
\hline
\end{tabular}

\section{S2. Vibrational analysis of 1 and F- binding complexes}

Vibrational analysis gives two important information about $\mathbf{1}$ and $\mathrm{F}^{-}$binding complexes 1-F: the ground state geometry of the molecules and the vibrational modes that correspond to the structural differences among 1, endo-1-F, and exo-1-F.

Vibrational frequencies are calculated by employing numerical command in Gaussian 09 with the ONIOM(B3LYP/6-31+G(d):B3LYP/3-21G) level of theory. Overall vibrations are transformed to IR spectra after calculating the vibrational modes. Figure S1-(a), (b), and (c) show the IR spectra of the 1, endo-1-F, and exo-1-F cases, respectively. 

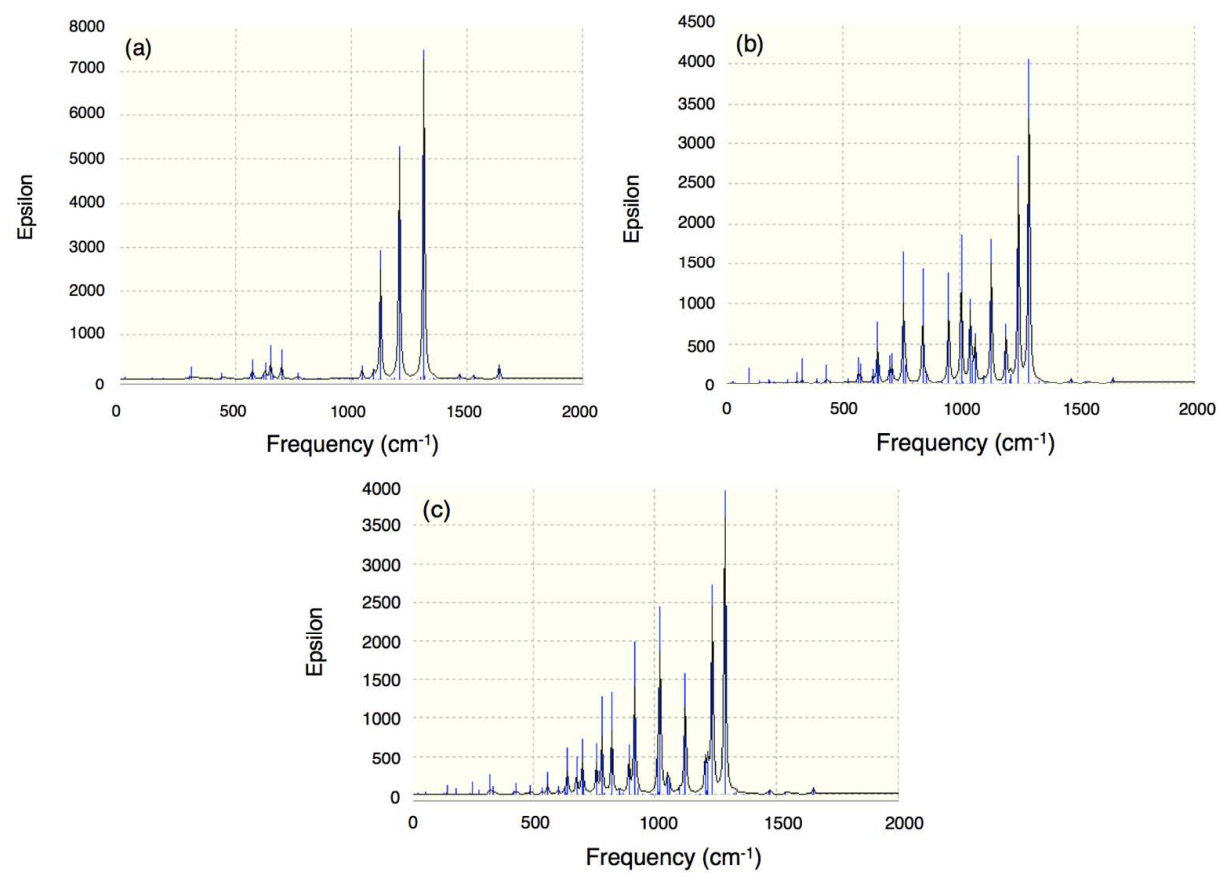

\section{Figure S1. Calculated IR spectra of bis(bora)calix[4]arene and its binding complexes with $\mathrm{F}^{-}$using ONIOM(B3LYP/6-31+G(d):B3LYP/3-21G) (a) 1 (b) endo-1-F (c) exo-1-F}

The optimized structures have no imaginary frequencies, which indicates the optimized structures of $\mathbf{1}$ and $\mathbf{1 - F}$ are the true minima on the ground state energy surface. To understand the major changes involved with vibrations while binding with fluoride, we compare the vibrational modes between endo-1-F and exo-1-F in the frequency domain from Figure S1.

Firstly, the epsilon values of the binding complexes are half of that of 1 . Although the general profile of the IR spectra remains similar for both binding complexes, some changes appear in the low frequency region and the $500-1200 \mathrm{~cm}^{-1}$ region. To further examine these changes, a comparison among the specific vibrations corresponding to the calculated peaks in these regions is conducted, as described below.

In the low frequency region, most of the vibrations are composed of displacements from the two wall benzenes (see Figure S4). There are two specific frequencies that correspond to the symmetrical displacement of the two wall benzenes in 1: moving in the same direction at $20 \mathrm{~cm}^{-1}$ and moving in the opposite direction toward the outer side at 40 
$\mathrm{cm}^{-1}$.

However, for binding complexes, these patterns have slightly changed with respect to the endo or exo-binding mode. For endo-1-F, a similar trend is observed, but decoupled motions originated by the fluoride are also observed. In detail, the vibrational mode in the same direction is observed at $22 \mathrm{~cm}^{-1}$ and the fluoride anion linked to the benzene moiety vibrates together. This additional vibration can affect the reduced mass of the vibrating molecule, resulting in the increased frequency. For exo-1-F, the vibration toward the outer side is hindered by the exo-bound fluoride anion, hence the calculated vibrations show the mixed motion of the inner space stretching in opposite/same directions. In the $200-300 \mathrm{~cm}^{-1}$ region, more complicated changes are observed from binding with the fluoride anion. Especially, a large displacement of the fluoride anion is observed at $265 \mathrm{~cm}^{-1}$ with wagging motion of the one benzene molecule in the lower rim. However, in the neutral case 1, this vibration is attributed to the in-plane vibration pattern of the two benzene walls at $252 \mathrm{~cm}^{-1}$. Therefore, in this case, the fluoride anion located between the two benzene rings breaks the symmetry of the motion, resulting in a decoupled motion of each benzene.

At $298 \mathrm{~cm}^{-1}, 1$ shows out-of-plane vibrations of the two oxygen-boron moieties with nearly identical intensities. However, endo-1-F shows asymmetric vibrations of those moieties in a slightly high frequency region at $305 \mathrm{~cm}^{-1}$. In this case, one oxygen-boron pair closest to the fluoride anion tends to move slowly compared to the neutral case because of the presence of the fluoride anion. Interestingly, this pattern is not observed in exo-1-F, which can be interpreted as a result of the blocking effect of the side-binding fluoride on oxygenboron stretching. A similar vibrational pattern in the opposite direction is observed at $307 \mathrm{~cm}^{-}$ ${ }^{1}$. Although this pattern is not observed in exo-1-F, endo-1-F shows a large displacement of the fluoride at $328 \mathrm{~cm}^{-1}$. This can be also understood as the blocking effect of the fluoride on the certain configurations of vibrations.

Some vibrations in the $820-840 \mathrm{~cm}^{-1}$ region contain weak vibrational modes of the fluoride, but these vibrations are considered as reactions from a large displacement of the boron atom. After $900 \mathrm{~cm}^{-1}$, no vibration motion of the fluoride is observed. 
In conclusion, the vibration analysis shows the ground state geometries and the structural characteristics of 1, endo-1-F, and exo-1-F.

\section{S3. Optimized geometries of various sensor motifs}

S3.1. Optimized geometries of 1-X (X: Cl-, Br-)

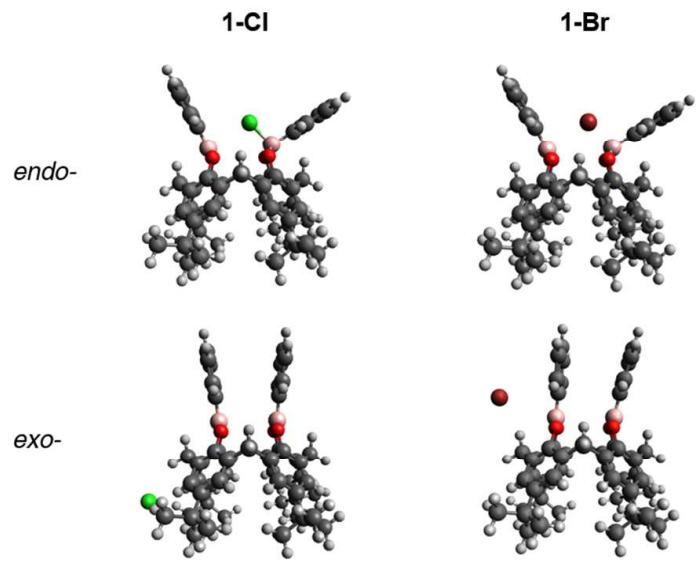

Figure S2. Optimized structures of 1-Cl and 1-Br complexes with both the endo- and exo- manners using ONIOM(B3LYP/6-31+G(d):B3LYP/3-21G) in the chloroform solution

It is worth denoting that exo-1-Cl (the chloride binding complex of 1-Cl via the exo-mode) is not regarded as an effective binding because it binds to the upper-rim part of 1-Cl.

S3.2. Optimized geometries of endo-XY-1F and exo-XY-1F (X, Y: B, P, N, and Al) 


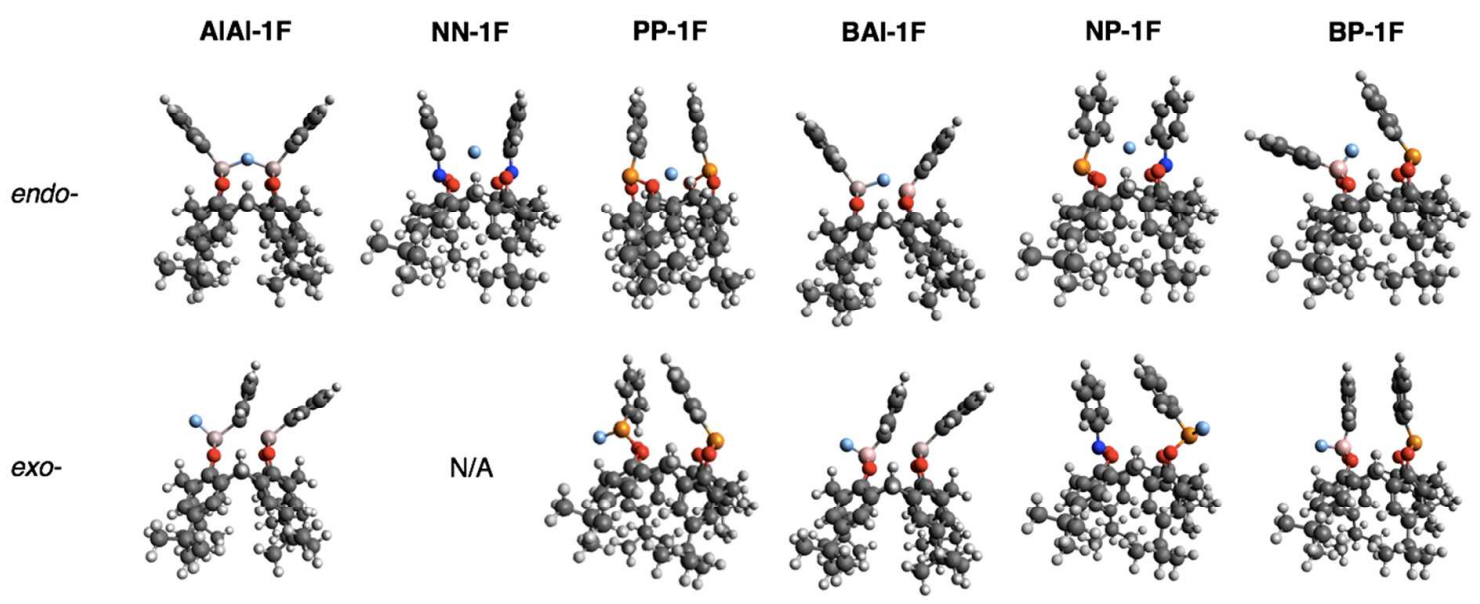

Figure S3. Optimized structures of endo-XY-1F and exo-XY-1F using ONIOM(B3LYP/6$31+G(d): B 3 L Y P / 3-21 G)$ in the gas phase

\section{S4. Fragment separation by Mulliken population in the frontier molecular orbital composition}

In order to perform the frontier molecular orbital analysis, we decompose $\mathbf{1}$ into four fragments: wall benzenes (green), boron atoms (yellow), oxygen atoms (blue), and the main 16-membered ring (red). The fragment decomposition of 1 gives an insight in terms of the nature of transitions at the electronic level. ${ }^{4}$ A detailed illustration is shown in Figure S4.

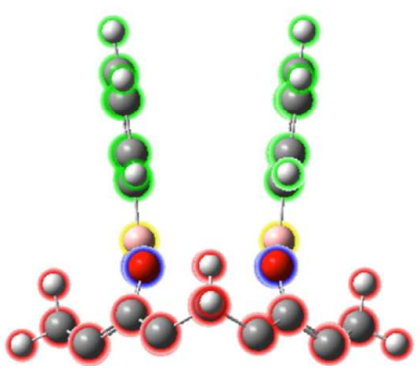

side-view

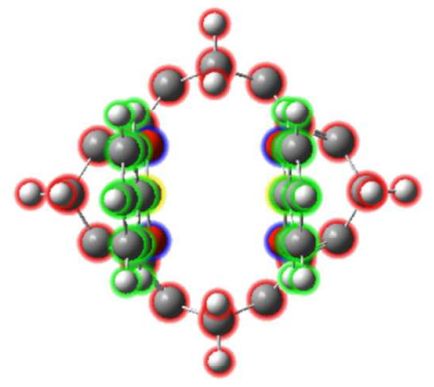

top-view

Figure S4. Mulliken fragments of 1 in the frontier MO decomposition (green: wall benzenes, yellow: boron atoms, blue: oxygen atoms, red: main ring) 


\section{S5. Additional TD-DFT calculation in the first excited state}

To validate the optimized structure in the $\mathrm{S} 1$ excited state, the bond lengths and angles of $\mathbf{1}$ in the excited state are calculated and compared with the ground state values of the molecule (see Table S2).

Table S2. Calculated geometrical properties of bis(bora)calix[4]arene in the ground and first excited states

(a) Bond lengths

\begin{tabular}{lccccccc}
\hline Bond length $(\AA)$ & $\mathrm{B} 50-\mathrm{O} 45$ & $\mathrm{~B} 50-\mathrm{O} 47$ & $\mathrm{O} 47-\mathrm{C} 24$ & $\mathrm{O} 45-\mathrm{C} 14$ & $\mathrm{C} 12-\mathrm{C} 9$ & $\mathrm{C} 14-\mathrm{C} 12$ & B50-C51 \\
\hline Ground state & 1.400 & 1.399 & 1.378 & 1.376 & 1.528 & 1.400 & 1.547 \\
Excited state & 1.404 & 1.412 & 1.402 & 1.399 & 1.528 & 1.417 & 1.554 \\
$\delta$ (difference) & 0.004 & 0.013 & 0.024 & 0.023 & 0.000 & 0.017 & 0.007 \\
\hline
\end{tabular}

(b) Bond angles

\begin{tabular}{lcc}
\hline Bond angle $\left(^{\circ}\right)$ & $\angle \mathrm{O} 45-\mathrm{B} 50-\mathrm{O} 47$ & $\angle \mathrm{O} 46-\mathrm{B} 49-\mathrm{O} 48$ \\
\hline Ground state & 121.87 & 121.87 \\
Excited state & 125.86 & 112.14 \\
$\delta$ (difference) & 3.99 & 9.73 \\
\hline
\end{tabular}

Also, the root mean square (RMS) value of overall bond distances is $1.38 \AA$ in the ground state and $1.40 \AA$ in the first excited state, indicating that the general structure remains similar. Figure S5 shows the TD-DFT spectra of $\mathbf{1}$ in the ground and excited states.

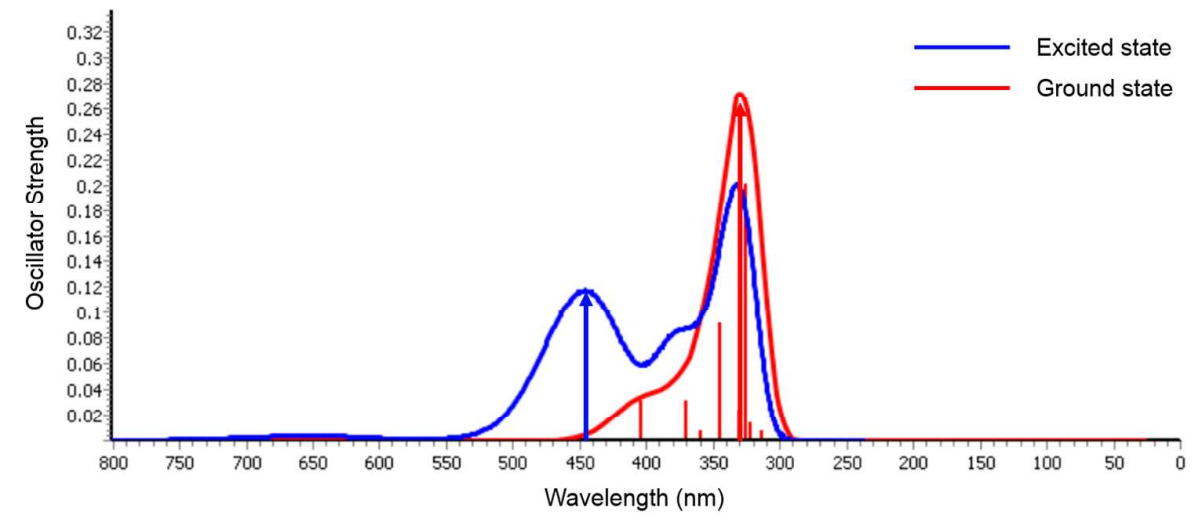

Figure S5. Calculated TD-DFT spectra of 1 in the ground and excited states with denoting important transition peaks as arrows using PBE1W/6-311G(d) 
Interestingly, both the ground and excited states spectra have a peak near $320 \mathrm{~nm}$, but they show different patterns in the longer wavelength region near $450 \mathrm{~nm}$. To elucidate the difference in this region, we assess the MO energy level and depict the MO diagrams of $\mathbf{1}$ in the ground and excited states (see Figure S6).
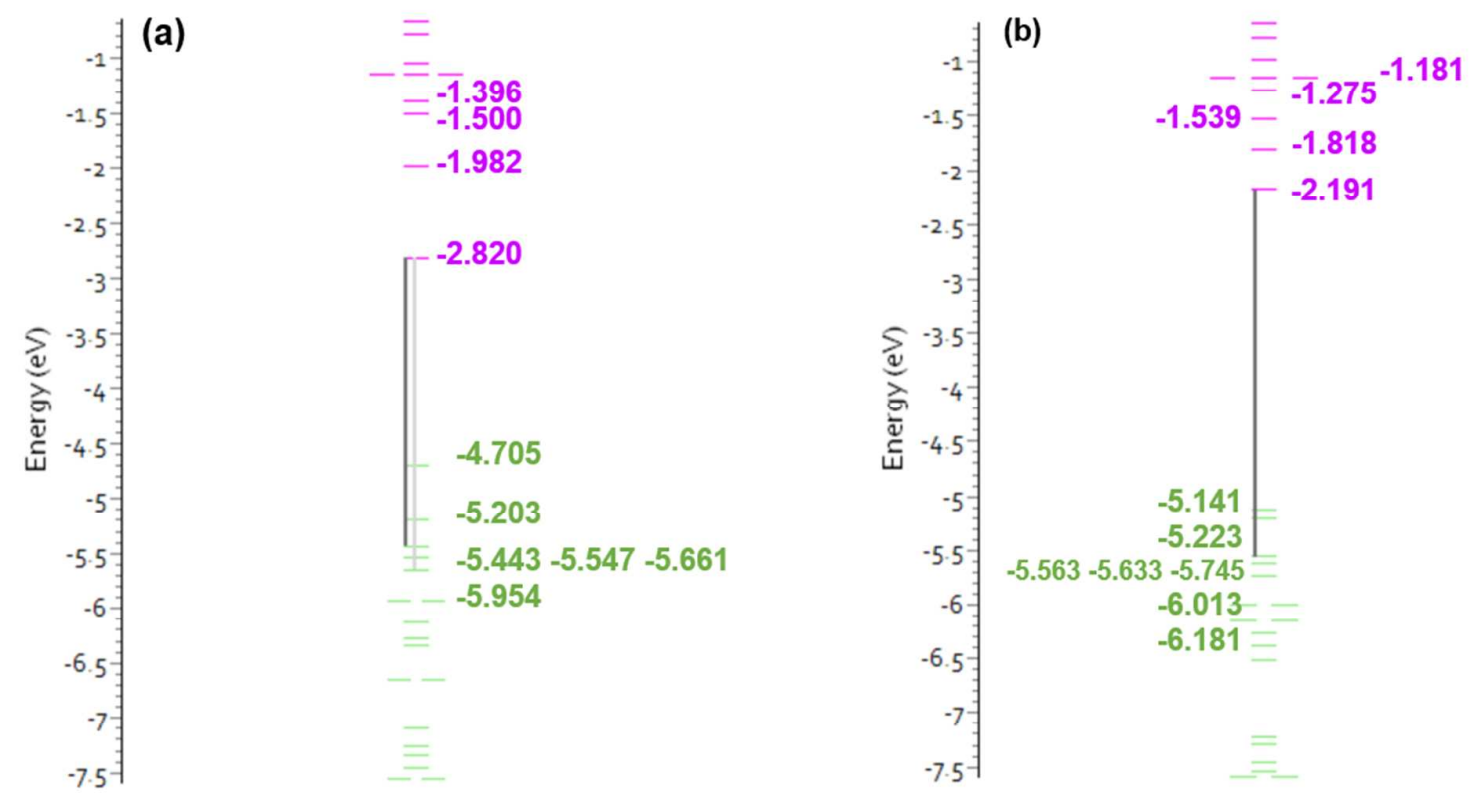

Figure S6. MO diagrams of bis(bora)calix[4]arene in the first excited and ground states with denoting energy values (eV) using PBE1W/6-311G(d):

(a) MO diagram in the excited state. (b) MO diagram in the ground state.

The neutral molecule, $\mathbf{1}$, is composed of 220 filled orbitals. Thus the HOMO is located at the $220^{\text {th }}$ MO and the LUMO at the $221^{\text {th }}$ MO. A comparison of Figure S6-(a) with (b) shows that the ground and excited states have similar orders of MO. When $\mathbf{1}$ is excited to the singlet state, the HOMO energy slightly increases and the LUMO energy slightly decreases, which result in a shift to a longer wavelength region (thus lower energy). This shift to a longer wavelength region is also consistent with the pattern from the simulated spectra. In Figure S5, the strong peak at $330 \mathrm{~nm}$ in the ground state, which is marked by a red arrow, originates from the transition of MO 218 to MO 221. However, this transition is shifted to $450 \mathrm{~nm}$ in the excited state, which is a mixed transition from 216 to 221 and 218 to 221 (marked by a blue arrow). 
To check the electron density in the lower rim, the LUMO and LUMO $+1\left(221^{\text {st }} \mathrm{MO}\right.$ and $\left.222^{\text {nd }} \mathrm{MO}\right)$ of $\mathbf{1}$, and the HOMO and HOMO-4 $\left(225^{\text {th }} \mathrm{MO}\right.$ and $\left.221^{\text {st }} \mathrm{MO}\right)$ of endo-1-F complex are depicted in Figure S7.

(a)
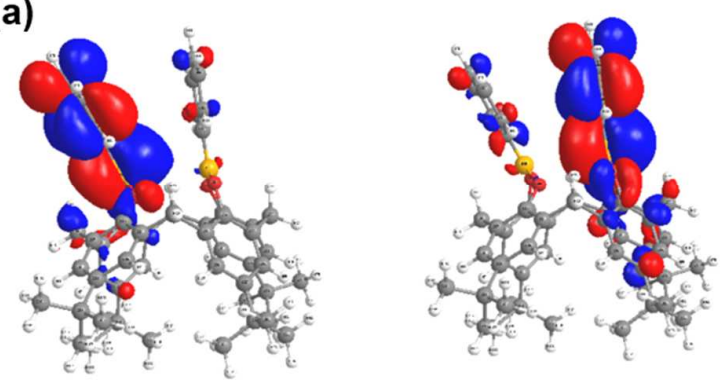

(b)
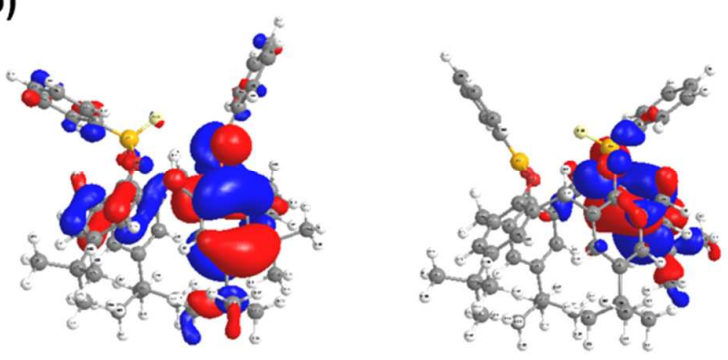

Figure S7. (a) The LUMO and LUMO+1 of 1 in the excited state (b) The HOMO and HOMO-4 of endo-1-F in the excited state using PBE1W/6-311G(d)

Figure S7-(a) shows the high electron density in the fluoride-binding region, and thus the change in calculated transitions while binding can be understood as an interaction between 1 and $\mathrm{F}^{-}$.

\section{S6. Additional TD-DFT calculation using CAM-B3LYP functional S6.1 Additional TD-DFT calculations using the CAM-B3LYP functional \\ TD-DFT calculations are also carried out on both 1 and 1-F using the CAM-B3LYP functional. ${ }^{5}$ Table S3 lists the vertical excitation of $\mathbf{1}$ with corresponding energy, oscillator strength, and major contributing transitions.}


Table S3. Vertical excitation of bis(bora)calix[4]arene in the ground state using CAM-B3LYP/6-311G(d)

\begin{tabular}{lclll}
\hline & \multicolumn{1}{c}{ Acalc } & Assignment (contribution) & $f_{\text {calc }}$ \\
\hline \multirow{2}{*}{ Band I } & $251.89 \mathrm{~nm}$ & $\begin{array}{l}\text { HOMO } \rightarrow \text { LUMO+1 (0.47) } \\
\text { HOMO } \rightarrow \text { LUMO }\end{array}$ & 0.0004 \\
& & $(0.16)$ & \\
Band II & $247.41 \mathrm{~nm}$ & HOMO-1 $\rightarrow$ LUMO+1 (0.45) & 0.0927
\end{tabular}

S6.2 Additional TD-DFT calculations using the PBE1W functional: Vertical excitation of 1 and endo-1-F in the S1 geometry

Table S4. Vertical excitation of 1 and $e n d o-1-F$ in the S1 (excited) state with corresponding energy and oscillator strength using PBE1W/6-311G(d)

(a) bis(bora)calix[4]arene

\begin{tabular}{lclc}
\hline & \multicolumn{1}{c}{ Acalc } & \multicolumn{1}{c}{ Assignment } & $f_{\text {calc }}$ \\
\hline Band A & $653.10 \mathrm{~nm}$ & HOMO $\rightarrow$ LUMO & 0.002 \\
Band B & $519.81 \mathrm{~nm}$ & HOMO-1 $\rightarrow$ LUMO & 0.001 \\
Band C & $383.21 \mathrm{~nm}$ & HOMO $\rightarrow$ LUMO+2 & 0.009 \\
Band D & $378.61 \mathrm{~nm}$ & HOMO-1 $\rightarrow$ LUMO+1 & 0.032 \\
\hline
\end{tabular}

(b) bis(bora)calix[4]arene with fluoride (the endo-binding)

\begin{tabular}{lrlr}
\hline & \multicolumn{1}{c}{$\Lambda_{\text {calc }}$} & \multicolumn{1}{c}{ Assignment } & $f_{\text {calc }}$ \\
\hline Band A' & $>700 \mathrm{~nm}$ & HOMO $\rightarrow$ LUMO & 0.000 \\
Band B' & $590.69 \mathrm{~nm}$ & HOMO-1 $\rightarrow$ LUMO & 0.001 \\
Band C' & $449.11 \mathrm{~nm}$ & HOMO $\rightarrow$ LUMO+2 & 0.007 \\
Band D' & $368.84 \mathrm{~nm}$ & HOMO-1 $\rightarrow$ LUMO+1 & 0.001 \\
\hline
\end{tabular}

\section{S7. Reverse $\mathbf{S 1} \rightarrow \mathbf{S 2}$ internal conversion calculation: $\Delta(\mathbf{S} 1-\mathbf{S} 2)$}

\section{S7.1. Schematic description}

To determine the origin of fluorescence from the higher excited states, the energy difference of the reverse internal conversion (or the thermal population) from the S1 to the S2 states is needed. ${ }^{6}$ Figure S8 shows the scheme of calculating the energy difference, $\Delta(\mathrm{S} 1-\mathrm{S} 2)$, at the first excited state. This schematic sketch of the potential energy surface (PES) is from Figure 5 in $\operatorname{Ref}(7)^{7}$ 


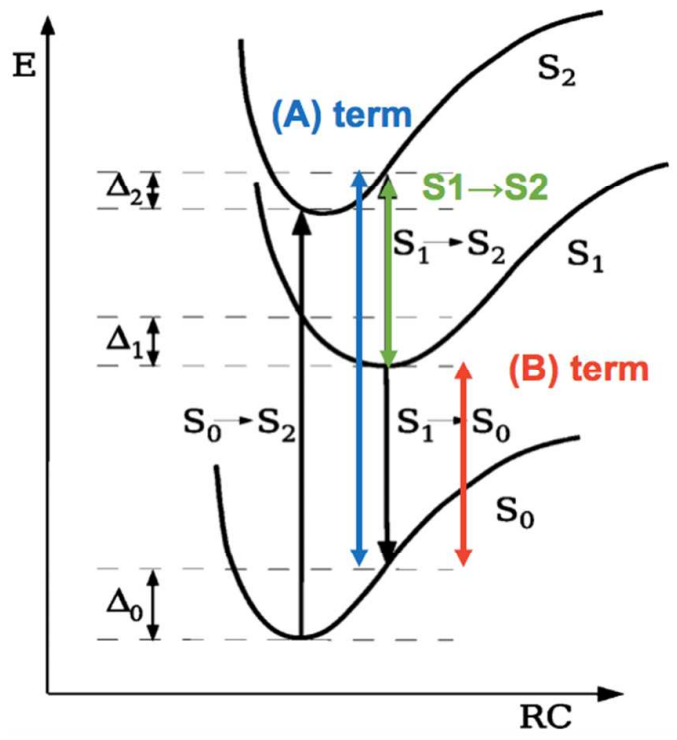

Figure S8. Schematic sketch of PES of the ground state (S0), first (S1) and second (S2) excited states of the selected system along the chosen reaction coordinate ${ }^{7}$

\section{S7.2. Results}

To calculate the energy difference $\Delta(\mathrm{S} 1-\mathrm{S} 2)$, one can decompose the (A) term, which corresponds to the energy difference between the S0 and S2 states of the optimized structure in the S1 state, as:

$$
\text { (A) term }=(\mathrm{B}) \text { term }+(\mathrm{S} 1 \rightarrow \mathrm{S} 2)
$$

Here, the (B) term corresponds to the emission energy from the S1 to the S0 states, which can be calculated as the TD-DFT excitation energy from the $\mathrm{S} 0$ to the $\mathrm{S} 1$ states at the structure optimized in the S1 state. Table S5 shows each term from the equation above and the resultant energy difference, implying that the reverse internal conversion may be very unlikely for 1 . 
Table S5. (A) term, (B) term and $\Delta(\mathrm{S} 1-\mathrm{S} 2)$ values from the simulation using PBE1W/6$311 G(d)$ level of theory

\begin{tabular}{|c|c|c|c|c|}
\hline Energy & (A) term & \multicolumn{2}{|c|}{ (B) term } & $\Delta(\mathbf{S 1 - S 2 )}$ \\
\hline Explanation & $\begin{array}{c}\text { Excitation Energy } \\
(\mathrm{S} 0 \rightarrow \mathrm{S} 2)\end{array}$ & $\begin{array}{c}\text { S1 Energy at S1 } \\
\text { optimized str. }\end{array}$ & $\begin{array}{c}\text { S0 Energy at S1 } \\
\text { optimized str. }\end{array}$ & $\begin{array}{c}\text { (A) term }- \\
(B) \text { term }\end{array}$ \\
\hline Value & $\begin{array}{c}2.3859 \\
(\mathrm{eV})\end{array}$ & $\begin{array}{c}-2522.822764 \\
(\text { Hartree })\end{array}$ & $\begin{array}{c}-2522.892612 \\
(\text { Hartree })\end{array}$ & $\begin{array}{c}3913.718761 \\
\left(\mathrm{~cm}^{-1}\right)\end{array}$ \\
\hline $\begin{array}{c}\text { Converted } \\
\text { Value }\end{array}$ & $\begin{array}{c}0.087680155 \\
(\text { Hartree })\end{array}$ & \multicolumn{2}{|c|}{$\begin{array}{c}(\mathrm{B}) \text { term }=0.06984794 \\
(\text { Hartree })\end{array}$} & $\begin{array}{c}0.017832215 \\
(\text { Hartree })\end{array}$ \\
\hline
\end{tabular}

\section{S8. UV-vis calculation of other potential fluoride sensors with group 15 atoms}

Figure S9 shows the simulated absorbance spectra of NN-1, PP-1, NP-1, and their endobinding and exo-binding complexes. The absorbance $\epsilon(v)$ of each spectrum is reproduced by the Harada-Nakanishi equation, ${ }^{8}$ which is followed by the equation below:

$$
\epsilon(\nu)=\sum_{i=1}^{N}\left(\frac{f_{i}}{3.483 \times 10^{-5} \sqrt{\pi} \sigma} \times \exp \left(-\left(\frac{\nu-\nu_{i}}{\sigma}\right)^{2}\right)\right)
$$

where $v$ is the excitation energy in $\mathrm{eV}, f_{i}$ is the oscillator strength from TD-DFT calculations, and $\sigma$ is the parameter chosen as $0.330 \mathrm{eV}$ to construct the Gaussian line shapes.
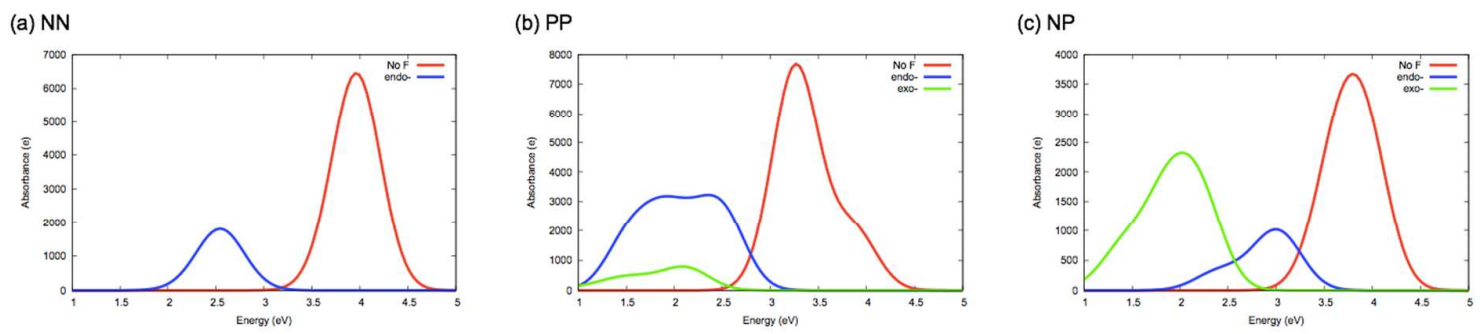

Figure S9. Calculated UV-vis spectrum of X-1, endo-X-1-F, and exo-X-1-F in the gas phase using PBE1W/6-311G(d) (the shape of spectrum is obtained by employing Gaussian spectrum with half width of $0.330 \mathrm{eV}$ ): (a) NN-1 (b) PP-1 (c) NP-1 


\section{References}

(1) Arimori, S.; Davidson, M. G.; Fyles, T. M.; Hibbert, T. G.; James, T. D.; KociokKo“hn, G. I. Synthesis and structural characterisation of the first bis(bora)calixarene: a selective, bidentate, fluorescent fluoride sensor. Chem. Commun. 2004, 14, 1640 - 1641.

(2) Bifulco, G.; Gomez-Paloma, L.; Riccio, R.; Gaeta, C.; Troisi, F.; Neri, P. Quantum mechanical calculations of conformationally relevant ${ }^{1} \mathrm{H}$ and ${ }^{13} \mathrm{C}$ NMR chemical shifts of calixarene systems. Org. Lett. 2005, 7, 5757-5760.

(3) Bifulco, G.; Riccio, R.; Gaeta, C.; Neri, P. Quantum Mechanical Calculations of Conformationally Relevant ${ }^{1} \mathrm{H}$ and ${ }^{13} \mathrm{C}$ NMR Chemical Shifts of N-, O-, and SSubstituted Calixarene Systems. Chem. Eur. J. 2007, 13, 7185-7194.

(4) Wang, J.; Bai, F. Q.; Xia, B. H.; Sun, L.; Zhang, H. X. Theoretical Understanding of Ruthenium(II) Based Fluoride Sensor Derived from 4,5-Bis(benzimidazol-2yl)imidazole (H3ImBzim) and Bipyridine: Electronic Structure and Binding Nature. $J$. Phys. Chem. A 2011, 10, 1985-1991.

(5) Yanai, T.; Tew, D. P.; Handy, N. C. A new hybrid exchange-correlation functional using the Coulomb-attenuating method (CAM-B3LYP). Chem. Phys. Lett. 2004, 393, $51-57$.

(6) Itoh, T. Fluorescence and phosphorescence from higher excited states of organic molecules. Chem. Rev. 2012, 112, 4541-4568.

(7) Dreuw, A. Influence of geometry relaxation on the energies of the S1 and S2 states of violaxanthin, zeaxanthin, and lutein. J. Phys. Chem. A 2006, 110, 4592-4599. 
(8) Harada, N.; Chen, S. M. L.; Nakanishi, K. Quantitative definition of exciton chirality and the distant effect in the exciton chirality method. J. Am. Chem. Soc. 1975, 97, 53455352. 